\title{
Surface modification toward luminescent and stable silica-coated quantum dots color filter
}

\author{
Bingxin Zhao ${ }^{1,2}$, Xiaoli Zhang ${ }^{1}$, Xue Bai ${ }^{1}$, Hongcheng Yang ${ }^{1,3}$, Shang $\mathrm{Li}^{1,3}$, Junjie Hao ${ }^{3}$, \\ Haochen Liu ${ }^{1}$, Rui Lu ${ }^{1}$, Bing Xu ${ }^{1}$, Liduo Wang ${ }^{1,2}$, Kai Wang ${ }^{1 *}$ and Xiao Wei Sun ${ }^{1 *}$
}

\begin{abstract}
A highly pixelated and luminescent silica-coated quantum dot color filter (QDCF) was achieved by surface conjugation with epoxy functional group. Epoxy-functionalized silica-coated quantum dots (QDs) can be thoroughly mixed with SU-8 photoresist up to 25 wt.\% without aggregation. The quantum yield (QY) of the silica-coated QDCF can be significantly improved from $19.3 \%$ to $36.5 \%$ after epoxy treatment. The pristine QDCF experienced a $40 \%$ QY decrease, while the epoxied silica-coated QDCF maintained its luminescence even after irradiation $\left(300 \mathrm{~mW} \mathrm{~cm}^{-2} @ 450 \mathrm{~nm}\right)$ for over 25 days. The well-controlled epoxy cap plays a critical role in attaining the ideal optical properties of the QDCF.
\end{abstract}

Keywords: quantum dot, silica coating, color filter, ligand exchange

\section{INTRODUCTION}

The BT.2020 color gamut standard has become a major research goal for the display industry [1]. The discovery and development of alternative highly efficient emitting materials with narrow bandgaps in the red-green-blue (RGB) spectral region is important and challenging [2]. Quantum dots (QDs) have been applied as promising emitting materials to enhance the display color gamut due to their unique properties, such as tunable bandgap, adjustable size, and controlled emission wavelength [3-6]. Generally, there are four types of QD applications in displays (including liquid crystal display (LCD) and micro light emitting diode (Micro-LED)), as summarized in Fig. S1. RGB pixels are normally obtained using traditional absorptive color filters (CFs), wherein the proper mixture of red and green QDs is excited with a blue LED chip. To achieve this, QDs can be embedded in packaged blue LED chips or tightly deposited on the surface of packaged chips (Fig. S1a and b); however, both these methods involve heat resistance issues. Ensuring an appropriate distance between the QD layers and packaged chips can overcome this problem (Fig. S1c and d). As shown in Fig. S1d, the traditional absorptive CFs are replaced with a wavelength-converting quantum dot color filter (QDCF), which can directly provide individual red and green pixels, with high color purity, efficient luminescence, wide-angle viewing, and a wide color gamut [3]. Therefore, the QDCF is critically important for newly emerging display techniques.

In preparing efficient QDCFs, fabricating proper QD substrates is a principal factor. Fully cured cross-linking photoresists exhibiting excellent mechanical properties [7] are a good choice for QD matrices. However, QDs normally possess different surface characteristics with a photoresist [8], resulting in significant phase segregation when directly mixed with a photoresist. This phase segregation dramatically hinders the application of QD/ photoresist hybrids. Extensive research efforts have been devoted to dispersing inorganic QDs in organic polymers to improve performance. Electrolyte salts can stabilize colloidal nanocrystals in solvents $[9,10]$. However, the presence of electrolytes in the polymer will dramatically compromise its physical properties. Specific surface modification through capping agents was directly employed with QDs to enhance colloidal stability in the polymer [11-14]. However, manipulating native surfacecapping ligands of QDs will quench the nanocrystal luminescence and thus decrease their shelf life [15]. Moreover, the QY of QDs is strongly affected by their dispersion in polymers, which is closely related to the

\footnotetext{
${ }^{1}$ Guangdong University Key Lab for Advanced Quantum Dot Displays and Lighting, Shenzhen Key Laboratory for Advanced Quantum Dot Displays and Lighting, Department of Electrical \& Electronic Engineering, Southern University of Science and Technology, Shenzhen 518055, China

${ }^{2}$ Department of Chemistry, Tsinghua University, Beijing 100084, China

${ }^{3}$ Shenzhen Planck Innovation Technologies Pte. Ltd., Shenzhen 518112, China

*Corresponding authors (emails: sunxw@sustc.edu.cn (Sun X); wangk@sustc.edu.cn (Wang K))
} 
surface trap states. Specifically, the cross-linking unit of the photoresist polymer is hydrolyzed (or degraded or broken) by an acid generated by a photoacid generator on the exposed region [16], which severely degrades the emission properties of QDs.

A common method for circumventing these issues is encapsulating the QDs with a hydrophilic silica shell, which is a basic strategy for protecting QDs from environmental degradation. The stability of the silica-coated QDs has been significantly improved in previous studies on $\mathrm{CdSe}-\mathrm{SiO}_{2}, \mathrm{CdSe} / \mathrm{ZnS}-\mathrm{SiO}_{2}, \mathrm{CdSe} / \mathrm{CdS} / \mathrm{ZnS}-\mathrm{SiO}_{2}$, and ${\mathrm{ZnSe}-\mathrm{SiO}_{2}}_{2}$ [17-22]. Generally, silica coating has been reported to decrease the QY [23], but increased QYs were achieved by Selvan's group [24] using a reverse microemulsion technique. In this approach, QDs were exposed to a silica precursor (tetraethyl orthosilicate, TEOS) in the presence of a surfactant. Rapid ligand exchange of the native surfactants with TEOS occurs, which is a critical step for incorporating QDs in a reverse micelle that forms a starting point for silica growth [25]. Therefore, a silica shell can prevent leakage of toxic $\mathrm{Cd}^{2+}$, enhance the chemical stability of the QDs [26,27], and promote surface conjugation with the ligand [15]. Moreover, it was reported that a silica shell can prolong the shelf life of QD devices: after long-term operation for $100 \mathrm{~h}$ at a driving current of $60 \mathrm{~mA}$, a silica QD-LED retained $88 \%$ of its initial QY, while a pristine QD-LED only retained 65\% of its initial value [28].

In this work, we prepared silica-coated QDs by a reverse microemulsion technique and investigated the interaction dynamics of QDs in an SU-8 photoresist. To fabricate a stable QDCF with high pixelation and luminescence, surface passivation of the silica-coated QDs in the photoresist was conducted using (3-glycidyloxypropyl) trimethoxysilane. For comparison, different samples are denoted as QDs, pristine silica-coated QDs, and epoxy silica-coated QDs. Ligand exchange at the silica surface involves replacing native - $\mathrm{OH}$ ligands with epoxy functional groups, which is conducive to obtaining photoresist-soluble silica-coated QDs. Therefore, after epoxy modification, silica-coated QDs can be dispersed uniformly in the matrix and are expected to be applied as stable and luminescent QDCFs.

\section{EXPERIMENTAL SECTION}

\section{Preparation of silica-coated QDs}

Unless specified otherwise, chemicals were purchased from Sigma-Aldrich and used without further purification. CdSe/ZnS QDs (denoted as QDs) were incorporated in silica spheres by a reverse microemulsion method; $10 \mathrm{~g}$ IGEPAL $^{\circledR}$ CO-520 surfactant, $3 \mathrm{~mL}$ TEOS, $640 \mu \mathrm{L}$ QDs/ methylbenzene $\left(50 \mathrm{mg} \mathrm{mL}^{-1}\right.$ ), and $3 \mathrm{~mL}$ ammonium hydroxide were added to $160 \mathrm{~mL}$ cyclohexane with a constant stirring speed of $500 \mathrm{rpm}$. The final mixed solution was stirred continuously for $24 \mathrm{~h}$ in dark.

\section{Synthesis of epoxied silica-coated QDs}

Ammonia solution $(30 \%)$ was added to an ethanol solution of silica QDs $\left(4 \mathrm{mg} \mathrm{mL}^{-1}\right)$ at room temperature (RT) to reach specific $\mathrm{pH}$ values. A molar ratio of 1:1 (3-glycidyloxypropyl) trimethoxysilane in chloroform solution (10\%) was added dropwise to the silica-coated QD suspension under vigorous stirring. After $24 \mathrm{~h}$, the epoxied silica-coated QDs were separated by centrifugation, washed with ethanol, and dispersed in propylene glycol monomethyl ether acetate (PGMEA).

\section{Fabrication of QDCF}

To compare the effects of silica coating and surface passivation on the QDCF, we prepared CdSe/ZnS QDs, pristine silica-coated QDs, and epoxied silica-coated QDs. These three types of QD were mixed with SU-8 3050 photoresist at the same concentration. Spin coating of SU8/QDs in PGMEA ( $v: v$ 1:1) was performed at $500 \mathrm{rpm}$ for $5 \mathrm{~s}$ and 3,000 rpm for $30 \mathrm{~s}$. Soft baking was performed for $9 \mathrm{~min}$ at $120^{\circ} \mathrm{C}$, followed by UV exposure at $365 \mathrm{~nm}$ for $15 \mathrm{~s}\left(300 \mathrm{~mW} \mathrm{~cm}^{-2}\right)$. Post-exposure baking was performed for $3 \mathrm{~min}$ at $100^{\circ} \mathrm{C}$. The samples were developed by immersion in SU-8 developer for $5 \mathrm{~min}$ to remove the unexposed areas, followed by rinsing with isopropylamine and drying with nitrogen gas.

\section{Characterization}

Transmission electron microscopy (TEM) was conducted with a Tecnai F30 microscope. Fourier transform infrared (FTIR) spectroscopy was performed using $\mathrm{KBr}$ tablets with a Perkin Elmer Frontier spectrometer (4,000$750 \mathrm{~cm}^{-1}$ ). Scanning electron microscopy (SEM) images were acquired with a field-emission scanning electron microscope (FE-SEM, JEOL JSM-7401F). Photoluminescence QY (PLQY) measurements were performed at RT with a C11347-12 (HAMAMATSU) absolute QY measurement system (excitation wavelength: $450 \mathrm{~nm}$ ). The optical output power of the blue LED (AISU 2835) for photoaging was measured using an ATA-500 Spectral Radiation Analyzer (EVERFINE Corporation) with an integrating sphere. Dividing the optical power by the sample area, we obtained the optical power density of photoaging. All optical measurements were performed at 
RT under ambient conditions.

\section{RESULTS AND DISCUSSION}

We successfully fabricated QDCF thin film with wellordered patterns via a process involving a typical photolithography technique, as shown in Fig. 1, where the silica-coated QDs are employed in this work. PGMEA was the developing agent for the SU-8 photoresist. As shown in Fig. 1, the QD/photoresist mixture was spincoated on the substrate, followed by UV exposure (365 nm, $15 \mathrm{~s})$ after soft baking. The masks formed the well-ordered patterns, such as circular, triangular, and square shapes with the same dimensions and spacing. After development, the non-irradiated part was removed, and the designed patterns were clearly and completely preserved. To obtain cemented QDCF thin film, hard baking is an essential stage. QDs are extremely sensitive to chemicals and thermal treatment during photolithography. The most viable strategy for protecting QDs from degrading environments is to physically encase them with chemically stable oxide phases, and the most widely used oxide for QD encapsulation is silica [28,29]. It was reported that silica coating improved the colloidal, chemical, and photostability of QDs and reduced the penetration speed of moisture and oxygen into the emission materials $[21,30,31]$. Therefore, silica coating is expected to preserve the QY during photolithography, as well as resist QD photodegradation.

To relieve phase segregation, which usually occurs in $\mathrm{QD} /$ photoresist hybrids, surface modification is adopted in this work. As far as we know, SU-8 is an epoxy-based negative photoresist, which can be conjugated with epoxy group. For silica-coated QDs, the residual hydroxyl group provided by ammonium during silica coating is condensation-cross-linkable and can be employed in the epoxy-capping reaction. Therefore, a homogeneous QD/ photoresist compound can be realized through surface decoration of the silica-coated QDs using epoxy ligands. Surface modification of the silica-coated QDs was performed using epoxy-containing silane, involving ligand exchange on the QD surfaces, as shown in Fig. 2. Replacing native $-\mathrm{OH}$ ligands with epoxy groups is conducive to obtaining photoresist-soluble silica-coated QDs.

To optimize the ligand exchange conditions, the effects of temperature and $\mathrm{pH}$ on the QY of silica-coated QDs were clarified in this work, as summarized in Table S1 (see Supplementary information). At RT $\left(20^{\circ} \mathrm{C}\right)$, the QY of silica-coated QDs increases from $46.6 \%$ to $56.6 \%$ as pH increases from 7 to 8.5 , while sharply drops to $42.9 \%$ as $\mathrm{pH}$ reaches 10 . Moreover, the QY is affected by the reaction temperature; for example, the QY decreases from $56.6 \%$ to $51.7 \%$ as the temperature increases from 20 to $45^{\circ} \mathrm{C}$ at $\mathrm{pH}$ 8.5. By comparison, the condensation reaction with hydroxyl groups can be easily conducted in a slightly basic environment, and the optimum $\mathrm{pH}$ is $\sim 8.5$.

After surface modification and ligand exchange at silica-coated QDs, FTIR spectra were recorded to examine the epoxy ligands, as shown in Fig. 3. The characteristic peak at $1,104 \mathrm{~cm}^{-1}$ is attributed to the $\mathrm{O}-\mathrm{Si}-\mathrm{O}$ stretching vibration [32], indicating that silica coating was successful. Peaks are observed at $3,435 \mathrm{~cm}^{-1}$ for the $-\mathrm{OH}$ vibration, $3,360 \mathrm{~cm}^{-1}$ for stretching $[33,34]$, and $1,640 \mathrm{~cm}^{-1}$ for in-plane bending [35]. Ligand exchange was conducted in a basic environment $(\mathrm{pH}$ 8.5) with ethanol and ammonia water. Hence, the peak at $3,435 \mathrm{~cm}^{-1}$ is ascribed to the residual solvents (ethanol, ammonia water) in the pristine silica-coated QDs. SU-8 3000 photoresists use cyclopentanone as the primary solvent [35]; hence, the peak at $3,435 \mathrm{~cm}^{-1}$ for the $-\mathrm{OH}$ group may be due to cyclopentanone. Both SU-8 and cyclopentanone contain $-\mathrm{CH}_{2-}$ bonds $\left(2,920 \mathrm{~cm}^{-1}\right)$. Therefore, the absorption peaks at $1,199 \mathrm{~cm}^{-1}$ for the epoxide group [36] and $2,920 \mathrm{~cm}^{-1}$ for $-\mathrm{CH}_{2}$ - indicate the presence of epoxy groups on the surface of silicacoated QDs.

The morphologies of epoxied silica-coated QDs were studied via TEM, as shown in Fig. 4. The monodisperse silica-coated QDs are about $50 \mathrm{~nm}$ in size (Fig. 4a). As shown in Fig. 4b, for epoxied silica-coated QDCF samples, the CdSe/ZnS core is speperated by silca coating;

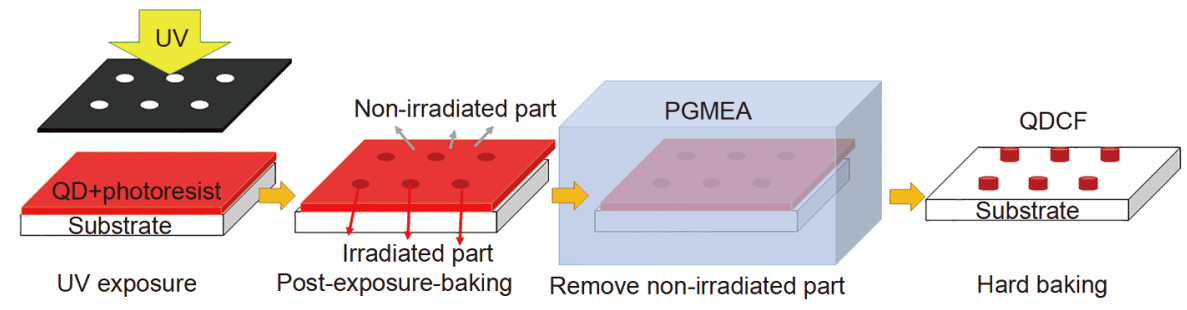

Figure 1 Schematic diagram of QDCF fabrication. 


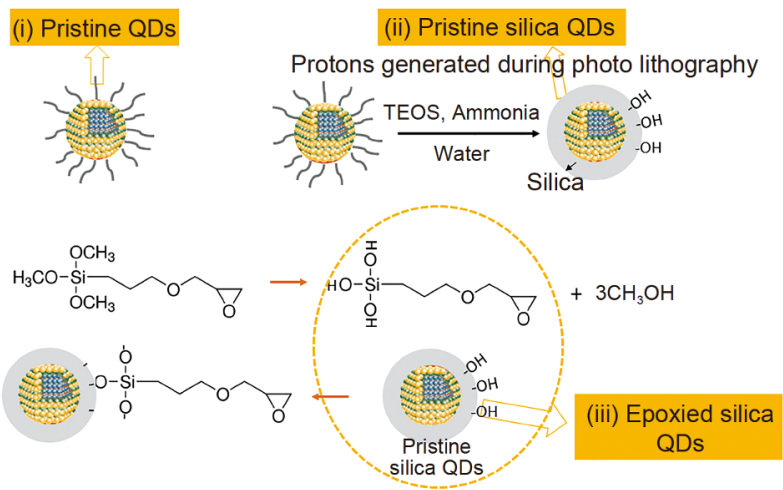

Figure 2 Schematic diagram of the ligand exchange process.

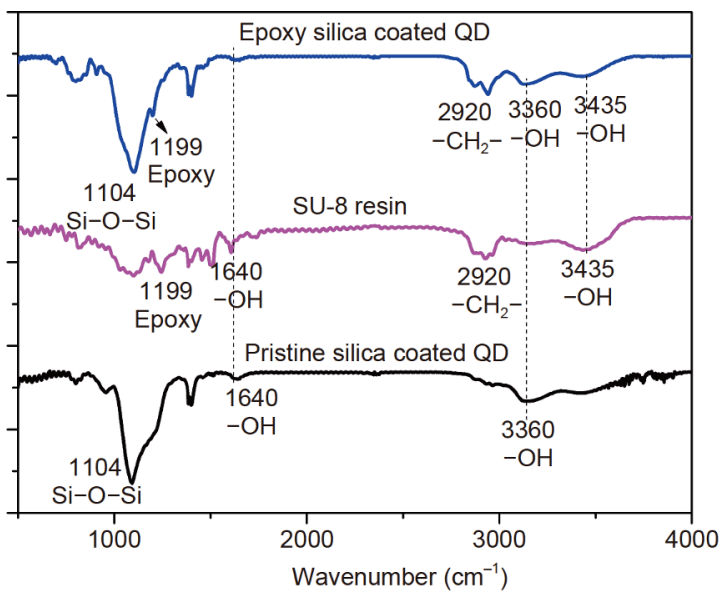

Figure 3 FTIR spectra of pristine silica-coated QDs, epoxied silicacoated QDs and SU-8 resin.

hence, the optical properties of the QDs are retained (Table S2). These epoxied silica-coated QDCF with high silica-coated QD concentration (25\%) is observed only at vertical direction, and some QDs are visually overlapped. Therefore, surface modification of silica-coated QDs by epoxy-capping effectively improves the solubility and dispersibility of QDs in the photoresist polymer.

To evaluate the effects of epoxy treatment on the developing process, the morphology evolution of pristine and epoxied silica-coated QDCFs is compared, as shown in Fig. S2 (see Supplementary information). The pristine silica-coated QDs are precipitated in PGMEA, while the corresponding epoxied samples can be easily dispersed in the solution. This illustrates that the epoxy functional groups are conducive to the dissolution of silica-coated QDs in PGMEA. The epoxied silica-coated QDCFs were then easily peeled off after the development, forming neat and orderly patterns on the substrate, as shown in

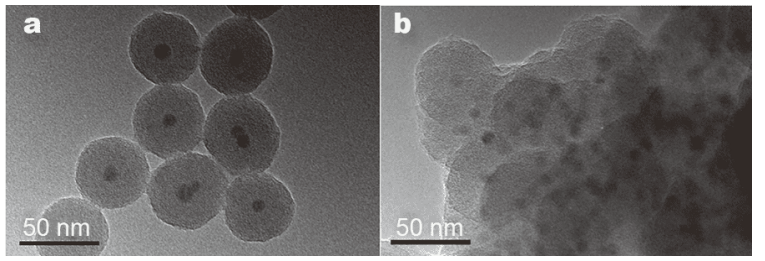

Figure 4 TEM images of pristine silica-coated QDs (a) and epoxied silica-coated QDs (25 wt.\%) in SU-8 photoresist (b).

Fig. S2e and f. Conversely, the pristine silica-coated QDCFs were extremely difficult to strip, leading to a disordered pattern (Fig. S2b and c). Therefore, epoxy modulation on silica-coated QDs is beneficial to the developing process and subsequent pattern fabrication.

In this work, we prepared color filters using epoxied silica-coated QDCF thin films to produce a badge of SUSTech (Southern University of Science and Technology), as shown in Fig. 5a. The badge pattern comprises a triangle, circle, and square in order, with the same size of $100 \mu \mathrm{m}$. From the SEM images, we can clearly see that the patterns are clear and neat after development, conforming to the original design.

To examine the effects of the QDCF fabricated using epoxied silica-coated QDs, PL emission spectra were recorded (Fig. S3). The QYs of different samples were compared, as summarized in Table S2. The QY of the asobtained QDs is only $11.4 \%$, which increases to $19.3 \%$ after silica coating and reaches $36.5 \%$ after surface modification. This indicates that the prepared QDCF exhibits an enhanced performance. The PL extraction enhancement is attributed to the silica protection, the uniform QD dispersion without aggregation, and the clean surface of the unexposed area after photolithography.

To study the photostability of the silica-coated QDCFs with and without epoxy treatment, the samples were subjected to aging tests under blue light at $300 \mathrm{~mW} \mathrm{~cm}^{-2}$ $@ 450 \mathrm{~nm}$. As shown in Fig. 6, the epoxied silica-coated QDCF presents a QY of $36.5 \%$ and shows a stable performance even after exposure in ambient air over 25 days. The PL intensity of the epoxied silica-coated QDCF gradually increases over time (photobrightening). Surface effects strongly influence the reactivity and optoelectronic properties of the QDs [37]: this external layer reversibly dissociates from and binds to the crystal surface, resulting in dynamic chemical formulas, surface reconstruction phenomena, and QD size reduction over time [38]. Jung et al. [39] reported 1.3 times of the initial PL intensity of RQDs@SiO ${ }_{2}$ after $32 \mathrm{~h}$ at $120^{\circ} \mathrm{C}$ under a tungsten halogen light. Ligands, amorphous silica, and metal complex lay- 

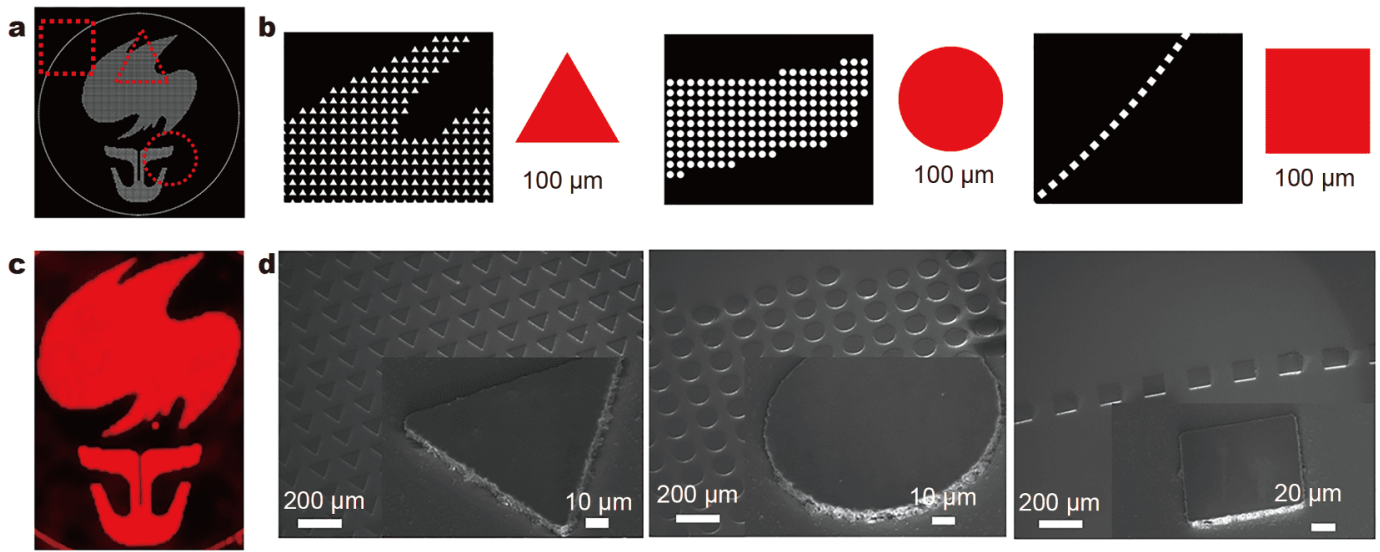

Figure 5 (a) Pattern mask originally designed for photolithography. (b) Mask dimensions. (c) Pixelated QDCF array molded on glass with $450 \mathrm{~nm}$ radiation. (d) SEM images of the epoxied silica-coated QDCF with well-ordered homogeneous shapes.

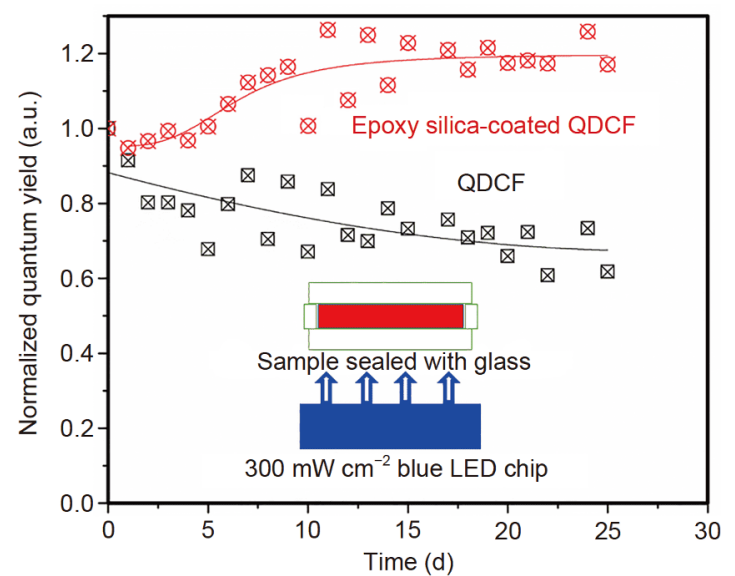

Figure 6 Photoaging of pristine QDCF and epoxied silica-coated QDCF (under $300 \mathrm{~mW} \mathrm{~cm}^{-2} 450 \mathrm{~nm}$ blue light) for over 25 days. The measured peak wavelengths are listed in Fig. S4. Note: the QDCF samples (overall size $5 \mathrm{~mm} \times 5 \mathrm{~mm}$ ) are used and placed $6 \mathrm{~mm}$ above the blue LED chip (overall size $2.8 \mathrm{~mm} \times 3.5 \mathrm{~mm}$ ).

ers affect the surface chemistry of epoxied silica-coated QDs. The photobrightening mechanisms might involve passivation of surface states and photochemical annealing $[39,40]$. Nevertheless, the QDCF based on pristine QDs exhibited a dramatic $40 \%$ decrease in QY. Therefore, introducing an epoxy cap in silica-coated QDs facilitates stable and efficient QDCF thin film fabrication.

\section{CONCLUSIONS}

In summary, we prepared highly pixelated and photostable QDCFs by photoetching. To obtain efficient QDCFs, QDs were passivated with silica and then decorated with epoxy via surface ligand exchange. Introducing epoxy functional groups on silica-coated QDs effectively enhances QD solubility in SU-8 photoresist. The epoxied silica-coated QDCF retained its initial PL and exhibited strong photostability against UV radiation for over 25 days.

Received 8 March 2019; accepted 30 April 2019; published online 22 May 2019

$1 \mathrm{Yu} \mathrm{D}$, Cao F, Gao Y, et al. Room-temperature ion-exchangemediated self-assembly toward formamidinium perovskite nanoplates with finely tunable, ultrapure green emissions for achieving Rec. 2020 displays. Adv Funct Mater, 2018, 28: 1800248

2 Meijerink A. Emerging substance class with narrow-band blue/ green-emitting rare earth phosphors for backlight display application. Sci China Mater, 2019, 62: 146-148

3 Lien JY, Chen CJ, Chiang RK, et al. High color-rendering warmwhite lamps using quantum-dot color conversion films. Opt Express, 2016, 24: A1021

4 Jun HK, Careem MA, Arof AK. Quantum dot-sensitized solar cells -perspective and recent developments: A review of Cd chalcogenide quantum dots as sensitizers. Renew Sustain Energy Rev, 2013, 22: 148-167

5 Liu SM, Guo HQ, Zhang ZH, et al. Characterization of CdSe and $\mathrm{CdSe} / \mathrm{CdS}$ core/shell nanoclusters synthesized in aqueous solution. Physica E-Low-dimensional Syst NanoStruct, 2000, 8: 174-178

6 Murray CB, Norris DJ, Bawendi MG. Synthesis and characterization of nearly monodisperse $\mathrm{CdE}$ ( $\mathrm{E}=$ sulfur, selenium, tellurium) semiconductor nanocrystallites. J Am Chem Soc, 1993, 115: 87068715

7 Martinez-Duarte R. SU-8 photolithography as a toolbox for carbon MEMS. Micromachines, 2014, 5: 766-782

8 Wei S, Zhu Z, Wang Z, et al. Improved photoluminescence quantum yield and stability of CdSe-TOP, CdSe-ODA-TOPO, CdSe/CdS and CdSe/EP nanocomposites. Mater Res Express, 2016, 3: 075904

9 Sun D, Sue HJ, Miyatake N. Optical properties of $\mathrm{ZnO}$ quantum dots in epoxy with controlled dispersion. J Phys Chem C, 2008, 112: 16002-16010

10 Dong X, Potter D, Erkey C. Synthesis of CuS nanoparticles in 
water-in-carbon dioxide microemulsions. Ind Eng Chem Res, 2002, 41: 4489-4493

11 Zillner E, Fengler S, Niyamakom P, et al. Role of ligand exchange at CdSe quantum dot layers for charge separation. J Phys Chem C, 2012, 116: 16747-16754

12 Anderson NC, Hendricks MP, Choi JJ, et al. Ligand exchange and the stoichiometry of metal chalcogenide nanocrystals: spectroscopic observation of facile metal-carboxylate displacement and binding. J Am Chem Soc, 2013, 135: 18536-18548

13 Beygi H, Sajjadi SA, Babakhani A, et al. Solution phase surface functionalization of $\mathrm{PbS}$ nanoparticles with organic ligands for single-step deposition of p-type layer of quantum dot solar cells. Appl Surf Sci, 2018, 459: 562-571

14 Nasri S, Bardajee GR, Bayat M. Solution phase surface functionalization of $\mathrm{PbS}$ nanoparticles with organic ligands for single-step deposition of p-type layer of quantum dot solar cells. Colloids Surfs B-Biointerfaces, 2018, 171: 544-552

15 Wolcott A, Gerion D, Visconte M, et al. Silica-coated CdTe quantum dots functionalized with thiols for bioconjugation to IgG proteins. J Phys Chem B, 2006, 110: 5779-5789

16 Campo A, Greiner C. SU-8: a photoresist for high-aspect-ratio and 3D submicron lithography. J Micromech Microeng, 2007, 17: R81R95

17 Selvan ST, Tan TT, Ying JY. Robust, non-cytotoxic, silica-coated CdSe quantum dots with efficient photoluminescence. Adv Mater, 2005, 17: 1620-1625

18 Zhang T, Stilwell JL, Gerion D, et al. Cellular effect of high doses of silica-coated quantum dot profiled with high throughput gene expression analysis and high content cellomics measurements. Nano Lett, 2006, 6: 800-808

19 Anderson BD, Wu WC, Tracy JB. Silica overcoating of CdSe/CdS core/shell quantum dot nanorods with controlled morphologies. Chem Mater, 2016, 28: 4945-4952

20 Zhao F, Zheng JG, Yang X, et al. Complex ZnO nanotree arrays with tunable top, stem and branch structures. Nanoscale, 2010, 2: 1674-1683

21 Jun S, Lee J, Jang E. Highly luminescent and photostable quantum dot-silica monolith and its application to light-emitting diodes. ACS Nano, 2013, 7: 1472-1477

22 Zhao B, Yao Y, Gao M, et al. Doped quantum dot@silica nanocomposites for white light-emitting diodes. Nanoscale, 2015, 7: $17231-17236$

23 Rogach AL, Nagesha D, Ostrander JW, et al. "Raisin bun"-type composite spheres of silica and semiconductor nanocrystals. Chem Mater, 2000, 12: 2676-2685

24 Selvan ST, Patra PK, Ang CY, et al. Synthesis of silica-coated semiconductor and magnetic quantum dots and their use in the imaging of live cells. Angew Chem Int Ed, 2007, 46: 2448-2452

25 Koole R, van Schooneveld MM, Hilhorst J, et al. On the incorporation mechanism of hydrophobic quantum dots in silica spheres by a reverse microemulsion method. Chem Mater, 2008, 20: 2503-2512

26 Modlitbová $\mathrm{P}$, Klepárník K, Farka Z, et al. Time-dependent growth of silica shells on CdTe quantum dots. Nanomaterials, 2018, 8: 439

27 Li C, Lu Z, Zhang Q, et al. Confined growth of CdSe quantum dots in colloidal mesoporous silica for multifunctional nanostructures. Sci China Mater, 2015, 58: 481-489

28 Jang EP, Jo JH, Kim MS, et al. Near-complete photoluminescence retention and improved stability of InP quantum dots after silica embedding for their application to on-chip-packaged light-emit- ting diodes. RSC Adv, 2018, 8: 10057-10063

29 Liu J, Song T, Yang Q, et al. Highly stable quantum dots with silica-poly(EGDMA-co-MAA) synergistic protection and the preliminary application in immunoassay. J Mater Chem B, 2013, 1: 1156-1163

30 Ziegler J, Xu S, Kucur E, et al. Silica-coated InP/ZnS nanocrystals as converter material in white LEDs. Adv Mater, 2008, 20: 40684073

31 Correa-Duarte MA, Giersig M, Liz-Marzán LM. Stabilization of CdS semiconductor nanoparticles against photodegradation by a silica coating procedure. Chem Phys Lett, 1998, 286: 497-501

32 Shen X, Wang Q, Liu Y, et al. Manganese phosphate self-assembled nanoparticle surface and its application for superoxide anion detection. Sci Rep, 2016, 6: 28989

33 Abdelrazek E, Abdelghany AM, Tarabiah AE. Characterization and physical properties of silver/PVA nano-composite. Res J Pharm Biol Chem Sci, 2012, 3: 448-459

34 Liu J, Tang J, Wang X, et al. Synthesis, characterization and curing properties of a novel cyclolinear phosphazene-based epoxy resin for halogen-free flame retardancy and high performance. RSC Adv, 2012, 2: 5789-5799

35 Robin CJ, Jonnalagadda KN. Effect of size and moisture on the mechanical behavior of SU-8 thin films. J Micromech Microeng, 2016, 26: 025020

36 Kang DY, Kim C, Park G, et al. Liquid immersion thermal crosslinking of 3D polymer nanopatterns for direct carbonisation with high structural integrity. Sci Rep, 2015, 5: 18185

37 Zherebetskyy D, Scheele M, Zhang Y, et al. Hydroxylation of the surface of $\mathrm{PbS}$ nanocrystals passivated with oleic acid. Science, 2014, 344: 1380-1384

38 Bertolotti F, Dirin DN, Ibáñez M, et al. Crystal symmetry breaking and vacancies in colloidal lead chalcogenide quantum dots. Nat Mater, 2016, 15: 987-994

39 Jung HS, Kim YJ, Cho J, et al. Silica-coated gradient alloy quantum dots with high luminescence for converter materials in white lightemitting diodes. RSC Adv, 2015, 5: 107585-107590

40 Osborne MA, Lee SF. Quantum dot photoluminescence activation and decay: dark, bright, and reversible populations in ZnS-capped CdSe nanocrystals. ACS Nano, 2011, 5: 8295-8304

Acknowledgements This work was supported by the National Key Research and Development Program of China administrated by the Ministry of Science and Technology of China (2016YFB0401702), the National Natural Science Foundation of China (61674074, 61704072 and 61405089), Shenzhen Innovation Project (JCYJ20160301113537474), Shenzhen Basic Research Project (JCYJ20170817112012493), Development and Reform Commission of Shenzhen Project ([2017]1395), Shenzhen Peacock Team Project (KQTD2016030111203005), Shenzhen Key Laboratory for Advanced Quantum Dot Displays and Lighting (ZDSYS201707281632549), Guangdong University Key Laboratory for Advanced Quantum Dot Displays and Lighting (2017KSYS007), Distinguished Young Scholar of National Natural Science Foundation of Guangdong (2017B030306010), Tianjin Zhonghuan Quantum Tech Co., Ltd. (18YFZCGX00580) and the start-up fund from Southern University of Science and Technology. This work was also supported by the Pico Center at SUSTech that received support from Presidential fund and Development and Reform Commission of Shenzhen Municipality, and China Postdoctoral Science Foundation Grant (2018M631443).

Author contributions Zhao B and Hao J conceived the experiments. 
Zhao B, Bai X and Lu R took part in detailed experiments. Zhao B and Zhang $\mathrm{X}$ prepared this manuscript. Liu H, Li S and Yang $\mathrm{H}$ were mainly responsible for the characterization in this work. Xu B provided chemicals used in this experiment. Wang L, Wang K and Sun X W carefully reviewed and modified this manuscript. All authors discussed on the results and commented on the manuscript.

Conflict of interest The authors declare no conflict of interest.

Supplementary information Supporting data are available in the online version of the paper.

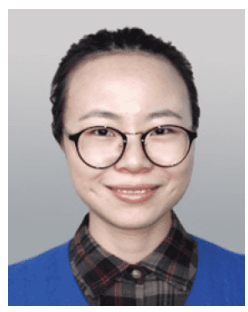

Bingxin Zhao received her $\mathrm{PhD}$ in materials science and engineering from Beijing University of Technology in 2017. In the same year, she carried out her postdoctoral research at Tsinghua University.

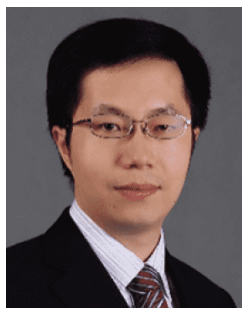

Kai Wang is currently an Associate Professor in the Department of Electrical and Electronic Engineering, Southern University of Science and Technology. He received $\mathrm{BSc}$ and $\mathrm{PhD}$ degrees from Huazhong University of Science and Technology in 2006 and 2011, respectively. His current research focuses on the semiconductor nanocrystal materials and their applications in optoelectronic devices, advanced displays, and solid-state lighting.

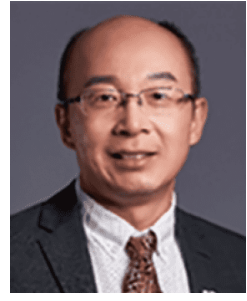

Xiao Wei Sun is currently a Chair Professor at the Southern University of Science and Technology, Shenzhen, China. He is also the Head of the Department of Electrical and Electronic Engineering. Before joining the Southern University of Science and Technology, he worked at Nanyang Technological University, Singapore, as a Full Professor. He was the Director of the Microelectronics Center at Nanyang Technological University. His main research is currently on semiconductor nanocrystals for power-saving high-quality displays and lighting. He has authored over 400 peer-reviewed publications and delivered numerous invited talks. His $\mathrm{H}$-index is 67 .

\section{通过表面针化实现稳定发光的硅包覆量子点 滤色片}

赵冰心 ${ }^{1,2}$, 张晓利 ${ }^{1}$, 白雪 ${ }^{1}$, 杨鸿成 ${ }^{1,3}$, 李尚 ${ }^{1,3}$, 郝俊㷊 ${ }^{3}$, 刘皓宸 ${ }^{1}$, 卢餐, 徐冰 ${ }^{1}$, 王立铎 ${ }^{1,2}$, 王恺 ${ }^{*}$, 孙小卫 ${ }^{*}$

摘要 通过表面连接环氧基团得到高亮度像素化的二氧化硅包覆 量子点滤色片(QDCF). 环氧基功能化的二氧化硅包覆量子点可以 与SU-8光刻胶均匀混合, 且混合比例高达 $25 \%$. 经环氧基处理之后, 二氧化硅包覆QDCF的量子产率 $(\mathrm{QY})$ 从 $19.3 \%$ 提高到 $36.5 \%$. 合成的 QDs经过 25 天的光照辐射( $300 \mathrm{~mW} \mathrm{~cm}^{-2} @ 450 \mathrm{~nm}$ ), QY较初始值降 低了 $65 \%$, 而环氧基二氧化硅包覆的QDCF没有明显衰减, 说明环氧 基调控对QDCF的光学性能起到了关键作用. 